\title{
SOMATOTIPO, FATORES DE RISCO E RAZÃO CINTURA- ESTATURA EM INDIVÍDUOS FISICAMENTE ATIVOS
}

\author{
SOMATOTYPES, RISK FACTORS AND WAIST-HEIGHT RATIO IN PHYSICALLY ACTIVE INDIVIDUALS
}

SOMATOTIPO, FACTORES DE RIESGO Y RAZÓN CINTURA-ESTATURA EN INDIVIDUOS

Artigo Original

Original Article

FISICAMENTE ACTIVOS

Anderson Henrique Souza de Almeida (Educador Físico)

Sarah Abrahão Gomes dos Santos² (Educadora Física)

Edil de Albuquerque Rodrigues Filho (Educador Físico)

Paulo Roberto Cavalcanti Carvalho (Educador Físico)

Gilmário Ricarte Batista ${ }^{3}$

(Educador Físico)

1. Universidade Federal de Pernambuco, Recife, PE, Brasil. 2. Universidade de Pernambuco, Recife, PE, Brasil.

3. Universidade Federal da Paraíba, João Pessoa, PB, Brasil.

\section{Correspondência:}

Rua Conde de Nassau, 357 B.

Várzea. Recife, PE, Brasil. 50740-630.

anderson_762@hotmail.com

\section{RESUMO}

Introdução: A relação entre o tipo físico e o risco cardiovascular vem sendo estudada em diversas populações do mundo. No Brasil, estudos que avaliam esta relação são escassos, principalmente quando se trata de indivíduos que praticam atividades físicas. Objetivo: Analisar a relação do somatotipo com fatores de risco cardiovascular e razão cintura-estatura (RCEst) em praticantes de atividade física. Métodos: Trata-se de uma pesquisa descritiva com delineamento transversal. A amostra foi constituída por 280 sujeitos, usuários da pista de Cooper da Universidade Federal de Pernambuco, na cidade de Recife, PE, Brasil. Para identificação do nível de atividade física, utilizou-se a versão curta do International Physical Activity Questionnaire (IPAQ). Os indivíduos classificados como muito ativos, ativos ou irregularmente ativos A ou B, foram incluídos na amostra. Excluíram-se os sujeitos inativos fisicamente. O somatotipo foi estimado através do protocolo antropométrico de Carter e Heath (1990). Determinaram-se como fatores de risco cardiovascular o tabagismo, o consumo de bebida alcoólica e a pressão arterial (PA). Empregou-se a estatística descritiva para caracterização da amostra, em seguida, utilizou-se a Análise de Variância Multivariada (MANOVA), com nível de significância de $p<0,05$. Resultados: Não foi identificada relação significativa para as variáveis tabagismo e consumo de bebida alcoólica. Na análise da PA (Pillai's trace $=0,082 ; F=8,187 ; p<0,05$ ) e da RCEst (Pillai's trace $=0,298 ; F=39,081 ; p<0,05$ ), verificou-se significância estatística com o somatotipo. Conclusão: O tipo físico foi positivo e significativamente relacionado com a PA e com a RCEst, demonstrando que esse indicador antropométrico pode ser utilizado para predizer precocemente o risco de desenvolvimento de doenças cardiovasculares.

Palavras-chave: doenças cardiovasculares, antropometria, somatotipos, atividade motora.

\section{ABSTRACT}

Introduction: The relationship between physical type and cardiovascular risk has been studied in several populations worldwide. In Brazil, studies evaluating this relationship are scarce, especially when physically active individuals are concerned. Objective: To analyze the relationship of the somatotype with cardiovascular risk factors and waist-height ratio (WHtR) in physically active individuals. Methods: This is a descriptive cross-sectional study. The sample consisted of 280 individuals, users of the jogging track at the Federal University of Pernambuco (UFPE) in Recife, PE, Brazil. To identify the level of physical activity, we used the short version of the International Physical Activity Questionnaire (IPAQ). Individuals classified as very active, active or irregularly active A or B were included in the sample. Physically inactive individuals were excluded from the analysis. The somatotype was estimated by the anthropometric protocol proposed by Carter and Heath (1990). We determined as cardiovascular risk factors smoking, alcohol consumption, and blood pressure (BP). We used descriptive statistics to characterize the sample, and then used a multivariate analysis of variance (MANOVA) with a significance level of $p<0.05$. Results: No significant relationship for the variables smoking and alcohol consumption. In the analysis of $B P$ (Pillai's trace $=0.082 ; F=8.187 ; p<0.05$ ) and WHtR (Pillai's trace $=0.298 ; F=39.081 ; p<0.05$ ) there was statistical significance for the somatotype. Conclusions: The physical type was positive and significantly related to BP and WHtR, showing that this anthropometric indicator can be used for early prediction of the risk of developing cardiovascular diseases.

Keywords: cardiovascular diseases, anthropometry, somatotypes, motor activity.

\section{RESUMEN}

Introducción: La relación entre el tipo de cuerpo y el riesgo cardiovascular, se ha estudiado en varias poblaciones de todo el mundo. En Brasil, los estudios de evaluación de esta relación son escasos, sobre todo cuando se trata de personas que practican actividades físicas. Objetivo: Analizar la relación entre el somatotipo y los factores de riesgo cardiovascular, y la razón cintura-estatura (RCEst) en practicantes de actividad física. Métodos: Se trata de un diseño transversal descriptivo. La muestra consistió en 280 sujetos, usuarios de una pista de Cooper de la Universidad Federal de Pernambuco, Recife, PE, Brasil. Para identificar el nivel de actividad física, se utilizó la versión corta del Internacional Physical Activity Questionnaire (IPAQ). Los individuos clasificados como muy activos, activos o irregularmente activos A o B se incluyeron en la muestra. Se excluyeron los sujetos físicamente inactivos. El somatotipo se estimó mediante el protocolo antropométrico de Carter y Heath (1990). Se determinaron como factores de riesgo cardiovascular el 
tabaquismo, el consumo de alcohol y la presión arterial (PA). Se utilizó estadística descriptiva para caracterización de la muestra y luego se utilizó el análisis multivariado de varianza (MANOVA), con un nivel de significación de p< 0,05. Resultados: Ninguna relación significativa fue identificada para las variables tabaquismo y consumo de alcohol. En el análisis de la PA (Pillai's trace $=0,082 ; F=8,187 ; p<0,05$ ) y RCEst (Pillai's trace $=0,298 ; F=39,081 ; p<0,05$ ), se verifico una significación estadística para el somatotipo. Conclusiones: El tipo físico fue positivo y significativamente relacionado con la PA y la RCEst, demostrando que este indicador antropométrico puede ser utilizado para predecir precozmente el riesgo de desarrollar enfermedad cardiovascular.

Palabras clave: enfermedades cardiovasculares, antropometría, somatotipos, actividad motora.

\section{INTRODUÇÃO}

As doenças cardiovasculares (DCV) constituem um grave problema de saúde pública', ocasionando nas últimas décadas cerca de 17 milhões de óbitos no mundo². No Brasil, estima-se que elas sejam responsáveis por quase $20 \%$ dos óbitos em sujeitos a partir dos vinte anos de idade ${ }^{3}$.

Essas doenças são de origem multifatorial e compartilham diversos fatores de risco como obesidade, tabagismo, consumo de álcool, inadequação alimentar, inatividade física, diabetes e hipertensão arterial ${ }^{4}$. Quanto maior o número de fatores de risco, maior a probabilidade de apresentar um evento cardiovascular ${ }^{5}$.

Mudanças no estilo de vida podem reduzir de forma significativa os fatores de risco cardiovascular ${ }^{6}$. Dentre essas mudanças, a prática regular de atividade física caracteriza-se como um comportamento importante, por agir na diminuição da gordura corporal ${ }^{7}$, prevenção e controle das doenças cardiovasculares ${ }^{8}$.

No estudo da distribuição da gordura corpórea, os métodos antropométricos têm sido frequentemente utilizados ${ }^{9}$. Nesse sentido, para uma melhor análise da forma corporal, utiliza-se a técnica antropométrica denominada somatotipo, que segundo Singh ${ }^{10}$, reflete uma visão global do corpo e transmite um significado de totalidade das características morfológicas do corpo humano.

O somatotipo tem assumido importância em estudos epidemiológicos, na medida em que permite associar os seus componentes (endomorfia, mesomorfia, ectomorfia) com características extremas da distribuição de gordura (central e periférica), tornando possível a identificação de grupos de risco ${ }^{11}$. Nesse contexto, acredita-se que elevados valores para o componente endomorfo, indicando grande quantidade de gordura corporal, possa estar associado ao risco cardiovascular ${ }^{12}$.

A relação do tipo físico com o risco cardiovascular vem sendo estudada em diversas populações do mundo ${ }^{13,14}$. No Brasil, estudos que avaliem esta relação são escassos, principalmente quando se trata de indivíduos que praticam atividades físicas. O presente estudo tem como objetivo analisar a relação do somatotipo com fatores de risco cardiovascular e razão cintura-estatura (RCEst) em indivíduos fisicamente ativos.

\section{MATERIAIS E MÉTODOS}

Trata-se de um estudo descritivo, do tipo transversal. A população foi constituída pelos usuários da pista de Cooper da Universidade Federal de Pernambuco (UFPE), na cidade de Recife, PE., Brasil. A UFPE desenvolve diversos projetos para incentivar os seus usuários à prática de atividade física, dentre eles destaca-se o Projeto Academia UFPE. De acordo com a coordenação desse projeto existiam 950 indivíduos inscritos nas práticas de atividades físicas (caminhada e corrida) no segundo semestre de 2010.

O processo de amostragem foi de forma não probabilística. Para o cálculo amostral foi considerado o erro amostral de 5\% e um intervalo de confiança de 95\%, perfazendo um número de 274 sujeitos. Por precaução, foram acrescentados 15\%, totalizando 315 indivíduos, como forma de garantir boa representatividade da amostra, em função de eventuais perdas no processo de coleta. A amostra final foi composta por 280 pessoas. Foram excluídos do estudo os indivíduos que recusaram à avaliação física, os sujeitos classificados como sedentários e os que responderam incorretamente ou que se negaram a responder qualquer uma das questões dos instrumentos aplicados.

O estudo recebeu a aprovação do Comitê de Ética em Pesquisa do Centro de Ciências da Saúde da Universidade Federal de Pernambuco, PE, Brasil, protocolado sob n. 103/10. Todos os participantes assinaram ao Termo de Consentimento Livre e Esclarecido, após serem informados sobre os procedimentos da pesquisa.

As avaliações foram realizadas no segundo semestre de 2010, em uma sala de apoio do Projeto Academia UFPE, durante o período matutino (das 6 h00 às 8h00), nas terças e quintas-feiras. Inicialmente, o estudo foi exposto com cartazes e faixas com explanação sobre os seus objetivos. Para identificação do nível de atividade física, utilizou-se a versão curta do International Physical Activity Questionare (IPAQ). Os indivíduos classificados como muito ativo, ativo ou irregularmente ativo A ou B, foram incluídos na amostra. Excluíram-se os sujeitos inativos fisicamente.

Foram analisadas variáveis comportamentais (tabagismo e consumo de bebida alcoólica), hemodinâmicas (pressão arterial sistólica e diastólica) e antropométricas (RCEst e somatotipo). Determinou-se como fatores de risco cardiovascular o tabagismo, o consumo de bebida alcoólica e a pressão arterial (PA). Em relação às variáveis tabagismo e consumo de bebida alcoólica, foi considerado em estado de risco o indivíduo que faz uso de qualquer quantia de cigarro diariamente ou que houver deixado de fumar por tempo mínimo de um ano, e aquele que ingere de forma habitual e diária bebida alcoólica, respectivamente ${ }^{15}$.

Para a PA considerou-se risco quando a pressão arterial sistólica $(P A S) \geq 140 \mathrm{mmHg}$ e pressão arterial diastólica (PAD) $\geq 90 \mathrm{mmHg}^{16}$. Quando as pressões sistólica e diastólica dos pesquisados situaram-se em categorias diferentes, utilizou-se para a classificação de risco a que estava acima do ponto de corte. A razão cintura-estatura (RCEst) foi classificada com base nos valores propostos por Pitanga e Lessa ${ }^{17}$ para a população brasileira, considerando-se risco quando maior ou igual a 0,53 para as mulheres e maior ou igual a 0,52 para os homens. Para efeito do estudo, as variáveis tabagismo, consumo de bebida alcoólica, PA e RCEst foram dicotomizadas em "sem risco" e "com risco".

A análise dos componentes do somatotipo foi realizada através da técnica antropométrica de Carter e Heath ${ }^{18}$. O somatotipo compõe-se de três componentes principais: a endomorfia, que representa a gordura corporal; a mesomorfia, que está relacionada à predominância da musculatura esquelética; e a ectomorfia, que corresponde à linearidade do corpo $^{18}$.

O peso corporal foi mensurado através de uma balança antropométrica portátil (marca Filizola, Brasil). A estatura foi medida por um 
estadiômetro portátil (marca Sanny, Brasil), de dois metros, escalonados em 0,1 cm. Também foram mensuradas quatro dobras cutâneas (tríceps, subescapular, suprailíaca e medial da perna), três circunferências (cintura, braço contraído e medial da perna) e dois epicôndilos (umeral e femural). As medidas das dobras cutâneas foram realizadas com um compasso Lange (Cambridge, Maryland, EUA), as circunferências com uma fita métrica (Cescorf, Brasil) e os epicôndilos através de um paquímetro antropométrico (Cescorf, Brasil) com aproximação de 0,1 mm.

A pressão arterial (PA) foi mensurada após cinco minutos de repouso na posição sentada, através de um esfigmomanômetro do tipo aneroide e estetoscópio, previamente calibrado e validado pelo INMETRO. Antes das aferições da PA, mensurou-se a circunferência do braço (CB) dos avaliados para determinar qual tipo de manguito seria utilizado. Se a $C B \geq 20 \mathrm{~cm}$ e $\leq 26 \mathrm{~cm}$, lançou-se mão do manguito de $10 \mathrm{~cm}$ de largura por $17 \mathrm{~cm}$ de comprimento. Para a $C B \geq 27 \mathrm{~cm}$ e $\leq 34$ $\mathrm{cm}$, foi utilizado o de $12 \mathrm{~cm}$ de largura por $23 \mathrm{~cm}$ de comprimento, e se $C B \geq 35 \mathrm{~cm}$ e $\leq 45 \mathrm{~cm}$, utilizou-se o de $16 \mathrm{~cm}$ de largura por $32 \mathrm{~cm}$ de comprimento ${ }^{19}$.

Todas as medições foram feitas no lado direito do corpo, em duplicata e realizadas por um único pesquisador para não comprometer a confiabilidade do estudo. A técnica de mensuração de erros foi utilizada de acordo com procedimentos aceitos e o erro de medida utilizado foi de no máximo $5 \%{ }^{20}$.

Empregou-se a estatística descritiva para caracterização da amostra. Para verificar a correlação entre as variáveis continuas, utilizou-se a correlação de Pearson. Utilizou-se a análise de variância multivariada (MANOVA) para verificar se os componentes do somatotipo diferem significativamente entre as variáveis independentes: tabagismo, consumo de bebida alcoólica, PA e RCEst. Quando verificada significância entre as comparações, aplicou-se o teste de post hoc de Tukey para localização das diferenças. O nível de significância estabelecido foi de 5\%. Utilizou-se, para as análises, o software estatístico SPSS, versão 16.0.

\section{RESULTADOS}

Na tabela 1, observa-se a análise descritiva da população em estudo. Em relação ao perfil do somatotipo, os valores para o componente endomorfo foram superiores ao mesomorfo e ectomorfo. Na análise da PAS e PAD, verificou-se que os indivíduos são normotensos. Os valores para a RCEst, indicaram que os sujeitos, de forma geral, não se encontraram na classificação de risco cardiovascular.

$\mathrm{Na}$ tabela 2, verificam-se as correlações dos componentes do somatotipo com a PAS e PAD e com a RCEst. Os resultados demostraram uma forte correlação entre os componentes do somatotipo e a RCEst.

A relação do somatotipo com os fatores de risco cardiovascular e com a RCEst são demonstradas na tabela 3. Não foram identificadas relações significativas para as variáveis tabagismo e consumo de bebida alcoólica. Na análise da PA (Pillai's trace $=0,082 ; F=8,187 ; p<0,05$ ) e da RCEst (Pillai's trace $=0,298 ; F=39,081 ; p<0,05$ ), verificou-se significância estatística com o somatotipo.

\section{DISCUSSÃO}

Estudos que verificam a influência dos padrões antropométricos sobre os fatores de risco cardiovasculares são importantes por avaliar quanto das modificações desses fatores, dependem de variações na composição corporal. Para tanto, na presente investigação, ao analisar a correlação da pressão arterial (sistólica e diastólica) e da razão cintura-estatura com os componentes do somatotipo, pôde-se verificar que o excesso de gordura corporal correlacionou-se positivamente com a elevação da pressão arterial.

A amostra do presente estudo consiste de indivíduos fisicamente
Tabela 1. Análise descritiva em média, desvio padrão, mínimo e máximo das mensurações antropométricas e hemodinâmicas.

\begin{tabular}{c|c|c}
\hline Variáveis & Média \pm DP & Mínimo-máximo \\
\hline Peso $(\mathrm{kg})$ & $69,4 \pm 13,5$ & $43-139$ \\
\hline Estatura $(\mathrm{m})$ & $1,64 \pm 0,09$ & $1,44-1,92$ \\
\hline Dobras cutâneas & & $5,7-59$ \\
\hline Tríceps $(\mathrm{mm})$ & $25,2 \pm 11,5$ & $6-62,3$ \\
\hline Subescapular $(\mathrm{mm})$ & $25,8 \pm 10,5$ & $5-68$ \\
\hline Supra ilíaca $(\mathrm{mm})$ & $30,6 \pm 11,5$ & $3-51$ \\
\hline Medial da perna $(\mathrm{mm})$ & $19,1 \pm 9,1$ & \\
\hline Circunferências & & $54,7-132,7$ \\
\hline Cintura (cm) & $84,6 \pm 12,2$ & $21,5-45,8$ \\
\hline Braço em contração (cm) & $31,1 \pm 3,7$ & $29-51,5$ \\
\hline Medial da perna (cm) & $36,6 \pm 3,2$ & $4,9-8$ \\
\hline Epicôndilos & & $7,5-12,2$ \\
\hline Umeral (cm) & $6,4 \pm 0,5$ & \\
\hline Femural (cm) & $9,7 \pm 1,0$ & $1,7-11,4$ \\
\hline Somatotipo & & $1,0-12$ \\
\hline Endomorfo & $7,1 \pm 1,7$ & $5,4-0,1$ \\
\hline Mesomorfo & $5,3 \pm 1,7$ & $85-185$ \\
\hline Ectomorfo & $1,3 \pm 1,2$ & $55-110$ \\
\hline PAS (mmHg) & $122,4 \pm 16$ & $32,9-76,7$ \\
\hline PAD (mmHg) & $77,0 \pm 10,5$ &
\end{tabular}

$\mathrm{DP}=$ Desvio padrão; $\mathrm{PAS}=$ Pressão arterial sistólica; $\mathrm{PAD}=$ Pressão arterial diastólica; $\mathrm{RCEst=} \mathrm{Razăo} \mathrm{cintura-estatura.}$

Tabela 2. Correlação entre os componentes do somatotipo e a PAS e PAD e entre a RCEst.

\begin{tabular}{c|c|c|c|c|c|c}
\hline \multirow{2}{*}{ Variáveis } & \multicolumn{2}{|c|}{ Endomorfo } & \multicolumn{2}{c|}{ Mesomorfo } & \multicolumn{2}{c}{ Ectomorfo } \\
\cline { 2 - 7 } & $\mathbf{r}$ & $\mathbf{p}$ & $\mathbf{r}$ & $\mathbf{p}$ & $\mathbf{r}$ & $\mathbf{p}$ \\
\hline PAS & 0,18 & $\mathrm{p}<0,01^{* *}$ & 0,36 & $\mathrm{p}<0,001^{*}$ & $-0,36$ & $\mathrm{p}<0,001^{*}$ \\
\hline PAD & 0,27 & $\mathrm{p}<0,001^{*}$ & 0,34 & $\mathrm{p}<0,001^{*}$ & $-0,41$ & $\mathrm{p}<0,001^{*}$ \\
\hline RCEst & 0,59 & $\mathrm{p}<0,001^{*}$ & 0,67 & $\mathrm{p}<0,001^{*}$ & $-0,76$ & $\mathrm{p}<0,001^{*}$ \\
\hline
\end{tabular}

${ }^{*} \mathrm{p}<0,001 ;{ }^{* *} \mathrm{p}<0,01 ; \mathrm{PAS}=$ Pressão arterial sistólica; PAD=Pressão arterial diastólica; RCEst=Razão cintura estatura.

Tabela 3. Relação do somatotipo com os fatores de risco cardiovascular e com a RCEst.

\begin{tabular}{c|c|c|c}
\hline Variáveis & $\begin{array}{c}\text { Endomorfo } \\
\text { Média (DP) }\end{array}$ & $\begin{array}{c}\text { Mesomorfo } \\
\text { Média (DP) }\end{array}$ & $\begin{array}{c}\text { Ectomorfo } \\
\text { Média (DP) }\end{array}$ \\
\hline Tabagismo & & & \\
\hline Com risco & $7,5(1,6)$ & $5,6(1,2)$ & $1,0(1,0)$ \\
\hline Sem risco & $7,1(1,7)$ & $5,3(1,7)$ & $1,3(1,2)$ \\
\hline Consumo de bebida alcoólica & & & \\
\hline Com risco & $7,0(1,7)$ & $5,2(1,5)$ & $1,3(1,1)$ \\
\hline Sem risco & $7,2(1,8)$ & $5,3(1,8)$ & $1,3(1,3)$ \\
\hline Pressão arterial & & & \\
\hline Com risco & $7,6(1,5)^{*}$ & $6,1(1,8)^{*}$ & $0,6(0,7)^{*}$ \\
\hline Sem risco & $7,0(1,8)$ & $5,1(1,6)$ & $1,4(1,3)$ \\
\hline RCEst & & & \\
\hline Com risco & $7,9(1,5)^{*}$ & $6,2(1,4)^{*}$ & $0,5(0,6)^{*}$ \\
\hline Sem risco & $6,5(1,6)$ & $4,6(1,6)$ & $1,8(1,3)$ \\
\hline
\end{tabular}

* $p<0,05 ;$ RCEst= Razão cintura estatura; DP= Desvio padrão.

ativos, assim espera-se que esse hábito esteja relacionado à boa saúde, como preconizado na literatura ${ }^{21}$. Contudo, os resultados apontam para a ocorrência de risco cardiovascular em parte da amostra, segundo alguns indicadores. Considerando que noutras pesquisas também se observa risco cardiovascular em pessoas habituadas à prática de atividade física, acredita-se muitas vezes, que apenas com o surgimento de risco cardiovascular, os indivíduos decidam assumir um estilo de vida ativo ${ }^{12}$.

Em estudos epidemiológicos, o hábito de fumar é considerado um fator de risco cardiovascular ${ }^{22}$, apresentando muitas vezes efeitos sobre a gordura corporal do indivíduo ${ }^{23,24}$. Klesges et al. ${ }^{25}$ ao estudarem a relação entre o uso de tabaco e o peso corporal, revelaram uma relação inversa, observando que a adiposidade tende a ser menor entre os indivíduos fumantes quando comparados aos não fumantes. Resultados distintos foram encontrados no presente 
estudo, assim como na investigação realizada por Clair et al. ${ }^{26}$, na qual não verificou-se significância estatística.

Na literatura, não são encontrados estudos que avaliem a relação dos componentes do somatotipo com o tabagismo, dificultando o aprofundamento dessa discussão. Todavia, a partir de outros indicadores antropométricos, estudos sugerem que o tabagismo tem um efeito em longo prazo sobre a adiposidade dos indivíduos ${ }^{23}$.

Os resultados do presente estudo também se assemelharam aos encontrados por Dallongeville et al. ${ }^{27}$, na medida em que não existe diferença na adiposidade entre sujeitos que fazem uso de bebida alcoólica. Segundo Castanheira et al. ${ }^{28}$, uma maior frequência na ingestão de bebida alcoólica não provoca efeito significativo sobre o acúmulo de gordura corporal. Outros estudos revelaram ainda associação negativa entre o consumo de bebida alcoólica e a adiposidade ${ }^{29}$. Nesse sentido, acredita-se que o alcoolista tenderia a reduzir sua ingestão alimentar, passando a suprir suas necessidades calóricas a partir da energia contida no álcool ${ }^{28}$.

Os indivíduos com risco cardiovascular segundo os níveis de pressão arterial apresentaram valores elevados para os componentes endomorfo e mesomorfo do somatotipo, quando comparados aos indivíduos sem risco. Esses resultados assemelham-se ao que nos traz o estudo de Herrera et al. ${ }^{14}$, no qual verificou-se que os sujeitos com pressão arterial sistólica e diastólica acima dos padrões normais, tinham o somatotipo semelhante ao de pessoas com outros fatores de risco cardiovascular, como infarto do miocárdio e doença cardíaca coronária.

Da mesma forma, os resultados trouxeram o componente ectomorfo significativamente mais alto nos sujeitos sem risco para a PA. Acredita-se assim, que os indivíduos que apresentam um perfil de risco cardiovascular são mais endomorfo e mesomorfo e menos ectomorfo do que aqueles com um perfil de menor risco cardiovascular ${ }^{13,14}$.

Considera-se a prática regular de atividade física, como uma estratégia para a prevenção e controle da hipertensão arterial e também para o tratamento de indivíduos hipertensos ${ }^{7}$. Dessa forma, é possível que esses benefícios para a pressão arterial, a partir da prática de atividade física, sucedam as mudanças no tipo físico, como a diminuição da endomorfia, enquanto componente de gordura.

\section{REFERÊNCIAS}

1. Simão M, Hayashida M, dos Santos CB, Cesarino EJ, Nogueira MS. Hypertension among undergraduate students from Lubango, Angola. Rev Lat Am Enfermagem. 2008;16(4):672-8.

2. Neumann Al, Martins IS, Marcopito LF, Araujo EA. Dietary patterns associated with risk factors for cardiovascular disease in a Brazilian city]. Rev Panam Salud Publica. 2007;22(5):329-39.

3. Guedes DP, Guedes JE, Barbosa DS, de Oliveira JA, Stanganelli LC. Cardiovascular risk factors in adolescents: biological and behavioral indicators. Arq Bras Cardiol. 2006;86(6):439-50.

4. Ricciardi R, Metter EJ, Cavanaugh EW, Ghambaryan A, Talbot LA. Predicting cardiovascular risk using measures of regional and total body fat. Appl Nurs Res. 2009;22(1):2-9.

5. Correia BR, Cavalcante E, Santos E. A prevalência de fatores de risco para doenças cardiovasculares em estudantes universitários. Rev Bras Clin Med. 2010;8(1):25-9.

6. Fernandes CE, Pinho-Neto JSL, Gebara OCE, Santos Filho RD, Pinto Neto AM, Pereira Filho AS, et al. I Diretriz Brasileira sobre Prevenção de Doenças Cardiovasculares em Mulheres Climatéricas e a Influência da Terapia de Reposição Hormonal (TRH) da Sociedade Brasileira de Cardiologia (SBC) e da Associação Brasileira do Climatério (SOBRAC). Arq Bras Cardiol. 2008;91(1Supl.1):1-23.

7. American College of Sports Medicine, Chodzko-Zajko WJ, Proctor DN, Fiatarone Singh MA, Minson CT, Nigg CR, et al. American College of Sports Medicine position stand. Exercise and physical activity for older adults. Med Sci Sports Exerc. 2009;41(7):1510-30.

8. Oldridge NB. Economic burden of physical inactivity: healthcare costs associated with cardiovascular disease. Eur J Cardiovasc Prev Rehabil. 2008;15(2):130-9.

9. Mirmiran P, Esmaillzadeh A, Azizi F. Detection of cardiovascular risk factors by anthropometric measures in Tehranian adults: receiver operating characteristic (ROC) curve analysis. Eur J Clin Nutr. 2004;58(8):1110-8.

10. Singh SP. Somatotype and disease: a review. Anthropologist. 2007;3:251-61.

11. Vasques CMS, Lopes VP, Seabra AFT, Silva SP, Maia JAR. Semelhança somatotipológica entre irmãos. Rev Bras Cineantropom Desempenho Hum. 2006;8(3):23-9.

12. Almeida AH, Santos SA, Castro PJ, Rizzo JA, Batista GR. Somatotype analysis of physically active individuals. J Sports Med Phys Fitness. 2013;53(3):268-73.

13. Kalichman L, Livshits G, Kobyliansky E. Association between somatotypes and blood pressure in an adult Chuvasha population. Ann Hum Biol. 2004;31(4):466-76.

14. Herrera H, Rebato E, Hernández R, Hernández-Valera Y, Alfonso-Sánchez MA. Relationship between somatotype and blood pressure in a group of institutionalized Venezuelan elders. Gerontology. 2004;50(4):223-9.

15. Carlini E, Galduróz J, Noto A, Nappo SI. I Levantamento domiciliar sobre o uso de drogas psicotrópicas no Brasil: estudo envolvendo as 107 maiores cidades do país. São Paulo: Cebrid/Unifesp; 2002.
A razão cintura-estatura tem sido proposta como uma alternativa no uso de indicadores antropométricos relacionados à saúde e, em muitos estudos, está fortemente associada aos diversos fatores de risco coronariano ${ }^{17,30}$. No presente estudo, ao analisar a relação dos componentes do somatotipo com a RCEst, enquanto indicador de risco cardiovascular, foi verificado que os indivíduos com risco são mais endomorfo e mesomorfo e menos ectomorfo do que aqueles sem risco.

Não existem outros estudos epidemiológicos que investiguem a RCEst e sua relação com o somatotipo. No entanto, esse indicador esteve presente na investigação de Flegal et al. ${ }^{30}$, que compararam o percentual de gordura corpórea com a RCEst, assinalando correlação significativa. Observa-se nesse estudo, assim como na presente pesquisa, que existe a relação da predominância de gordura corporal com a RCEst.

A presente investigação apresenta um delineamento transversal, portanto não nos permite apontar relações de causalidade entre as variáveis investigadas e o tipo físico. Outra fragilidade refere-se à determinação do nível de atividade física, mensurado através do auto relato, e, portanto, dependente da precisão da lembrança do avaliado. No entanto pode-se considerar essa pesquisa como um importante ponto de partida nessa área epidemiológica, uma vez que não foram encontrados estudos com a relação entre o somatotipo e o tabagismo, o consumo de bebida alcoólica e a RCEst.

\section{CONCLUSÕES}

O somatotipo dos praticantes de atividade física em estudo demostrou altos valores para o componente endomorfo e mesomorfo e baixos índices para ectomorfia, indicando que essa população encontra-se com alta predominância de gordura corporal. Verificou-se também que o tipo físico foi positivo e significativamente relacionado com a PA e com a RCEst, indicadores de risco cardiovascular, demostrando que esse indicador antropométrico pode ser utilizado para predizer, precocemente, o risco para o desenvolvimento de doenças cardiovasculares.

Todos os autores declararam não haver qualquer potencial conflito de interesses referente a este artigo.

16. Sociedade Brasileira de Cardiologia-SBC, Sociedade Brasileira de Hipertensão-SBH, Sociedade Brasileira de Nefrologia-SBN. V Brazilian Guidelines in Arterial Hypertension. Arq Bras Cardiol. 2007;89(3):e24-79

17. Pitanga FJG, Lessa I. Indicadores antropométricos de obesidade como discriminadores de risco coronariano elevado em mulheres. Rev Bras Cineantropom Desempenho Hum. 2006;8(1):14-21.

18. Carter JE, Heath HB. Somatotyping-development and application. Cambridge: University Press; 1990

19. Sociedade Brasileira de Cardiologia, Sociedade Brasileira de Hipertensão, Sociedade Brasileira de Nefrologia. VI Brazilian Guidelines on Hypertension. Arq Bras Cardiol. 2010;95(1 Suppl):1-51.

20. Malina RM. Anthropometry. Champaign: Human Kinetics; 1995.

21. Shibata A, Oka K, Nakamura Y, Muraoka I. Recommended level of physical activity and health-related quality of life among Japanese adults. Health Qual Life Outcomes. 2007;5:64.

22. Halperin RO, Gaziano JM, Sesso HD. Smoking and the risk of incident hypertension in middle-aged and older men. Am J Hypertens. 2008;21(2):148-52.

23. Xu F, Yin XM, Wang Y. The association between amount of cigarettes smoked and overweight, central obesity among Chinese adults in Nanjing, China. Asia Pac J Clin Nutr. 2007;16(2):240-7.

24. Sun K, Liu J, Ning G. Active smoking and risk of metabolic syndrome: a meta-analysis of prospective studies. PLoS One. 2012;7(10):e47791.

25. Klesges RC, Ward KD, Ray JW, Cutter G, Jacobs DR Jr, Wagenknecht LE. The prospective relationships between smoking and weight in a young, biracial cohort: the Coronary Artery Risk Development in Young Adults Study. J Consult Clin Psychol. 1998;66(6):987-93.

26. Clair C, Chiolero A, Faeh D, Cornuz J, Marques-Vidal P, Paccaud F, et al. Dose-dependent positive association between cigarette smoking, abdominal obesity and body fat: cross-sectional data from a population-based survey. BMC Public Health. 2011;11:23.

27. Dallongeville J, Marécaux N, Ducimetière P, Ferrières J, Arveiler D, Bingham A, et al. Influence of alcoho consumption and various beverages on waist girth and waist-to-hip ratio in a sample of French men and women. Int J Obes Relat Metab Disord. 1998;22(12):1178-83.

28. Castanheira M, Olinto MT, Gigante DP. Socio-demographic and lifestyle factors associated with abdominal fat distribution in adults: a population-based survey in Southern Brazil. Cad Saude Publica. 2003;19(Suppl 1):S55-65

29. Nishioji K, Sumida Y, Kamaguchi M, Mochizuki N, Kobayashi M, Nishimura T, et al. Prevalence of and risk factors for non-alcoholic fatty liver disease in a non-obese Japanese population, 2011-2012. J Gastroenterol. 2015;50(1):95-108.

30. Flegal KM, Shepherd JA, Looker AC, Graubard BI, Borrud LG, Ogden CL, et al. Comparisons of percentage body fat, body mass index, waist circumference, and waist-stature ratio in adults. Am J Clin Nutr. 2009;89(2):500-8. 